\title{
Using a pessary during radiotherapy in reducible pelvic organ prolapse and vaginal cancer: a case report and review of the literature
}

\author{
Melis Gultekin, MD!, Caglayan Selenge Beduk Esen, MD!, Utku Akgor, MD², Mehmet Coskun Salman, MD², \\ Murat Tuncel, MD³, Mustafa Ozmen, MD4, Ferah Yildiz, MD' \\ 'Department of Radiation Oncology, Hacettepe University Medical School, Ankara, Turkey, ${ }^{2}$ Department of Obstetrics and Gynecology, \\ Division of Gynecologic Oncology, Faculty of Medicine, Hacettepe University, Ankara, Turkey, ${ }^{3}$ Department of Nuclear Medicine, Hacettepe \\ University, Faculty of Medicine, Ankara, Turkey, ${ }^{4}$ Department of Radiology, Faculty of Medicine, Hacettepe University, Ankara, Turkey
}

\begin{abstract}
Purpose: Primary vaginal cancer and pelvic organ prolapse (POP) combination is extremely rare. Although definitive chemoradiotherapy (CRT) and brachytherapy (BT) is the standard treatment for advanced stage primary vaginal cancer, there is a limited data about the treatment of primary vaginal cancer combined with POP due to its rarity. In addition, radiotherapy (RT) process may be difficult in these cases and often result in more toxicity.

Case presentation: In this case report, we present a 77-year-old woman with a diagnosis of primary vaginal cancer associated with POP, who was treated with definitive CRT using a pessary to restore vaginal anatomy for optimal radiation. Following CRT, complete response was observed and vaginal cuff BT was performed. The patient tolerated the treatment very well and is still alive without disease at 10-months follow-up.

Conclusions: Combined primary vaginal cancer and POP is an extremely rare clinical entity, with only a few cases reported in the literature. When applying CRT in these cases, critical organ doses may be higher than expected due to the downward descent of the pelvic organs. Especially in elderly patients, a pessary can be used as a non-surgical procedure to restore the anatomy for symptom relief during definitive CRT. Additionally, it allows tumors to be targeted more precisely.

J Contemp Brachytherapy 2020; 12, 2: 175-180 DOl: https://doi.org/10.5114/jcb.2020.92997
\end{abstract}

Key words: primary vaginal cancer, pelvic organ prolapse, pessary, radiotherapy, brachytherapy.

\section{Purpose}

Primary vaginal cancers are rare, comprising 1-2\% of all gynecological malignancies and mostly seen at the age of 60-70 years [1]. These tumors usually occur in the upper one-third of the vagina, and squamous cell carcinoma is the most common histological type. Similar to vaginal cancer, pelvic organ prolapse (POP) has a peak prevalence of $5.1 \%$ in women aged $60-69$ years [2]. Vaginal trauma, chronic irritation, and inflammation of the vaginal mucosa can cause primary vaginal cancer in patients with POP [3]. However, a combination of POP and primary vaginal or cervical cancer is an extremely rare entity $[4,5,6,7,8,9,10,11,12,13,14,15,16,17,18,19]$.

Multidisciplinary management is necessary for optimal treatment of primary vaginal cancer. While surgery can be performed in early stage disease, definitive chemoradiotherapy (CRT) is the preferred treatment for the advanced stage. In this case, we report a 77-year-old woman with a diagnosis of primary vaginal cancer associated with a reducible POP. She was treated with definitive CRT and brachytherapy (BT) after using a pessary to restore vaginal anatomy for optimal radiation.

\section{Case report}

A 77-year-old women, gravida 4, parity 4, presented with a 7-month history of vaginal bleeding. She had a clinical history of total abdominal hysterectomy due to multiple uterine leiomyomas 40 years ago and she had vaginal vault prolapse for 13 years with no history of pessary use. Her medical history included hypertension and ischemic heart disease. Physical examination revealed manually reducible stage 4 vaginal vault prolapse according to the POP quantification system [20], with an infiltrating 1-2 $\mathrm{cm}$ mass between the upper and middle third of the posterior vaginal wall. There was no para- 


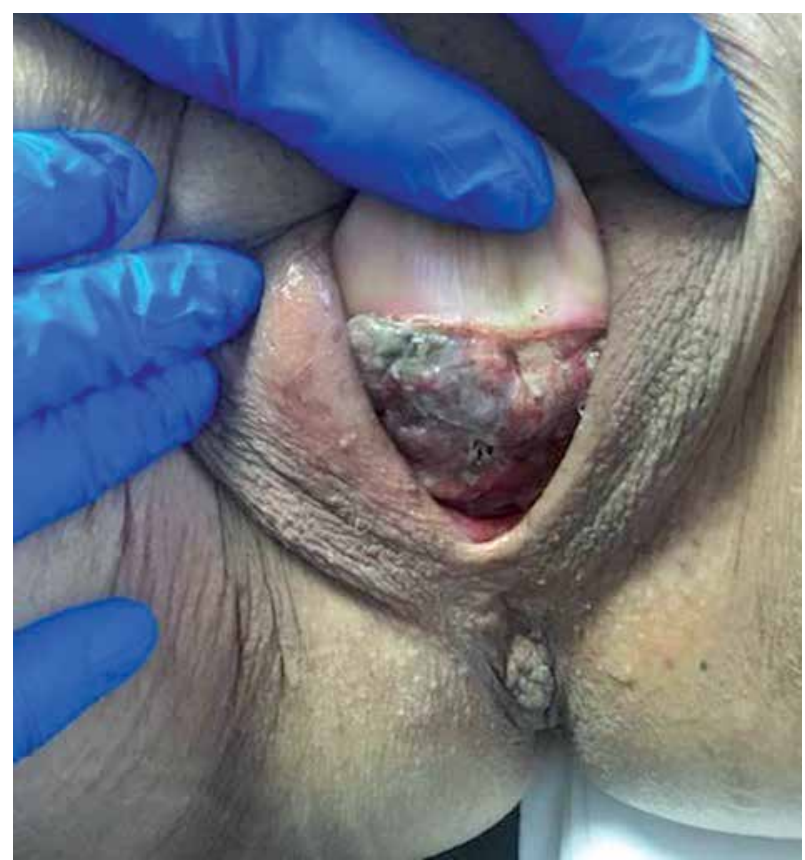

Fig. 1. Pre-treatment appearance of vaginal vault prolapse with an infiltrating mass metrial invasion, pelvic wall involvement, or inguinal lymph node metastases (Figure 1).

A magnetic resonance imaging (MRI) scan revealed tricompartmental (cystocele, enterocele, and vaginal vault) POP, but no visible tumor (Figure 2). Positron emission tomography/computed tomography (PET/CT) revealed high F-18 fluorodeoxyglucose (FDG) uptake in the primary vaginal mass $\left(\mathrm{SUV}_{\max }, 16.37\right)$ without any evidence of metastases to the lymph nodes or other organs (Figure 3). Incisional biopsy from the lesion was reported as squamous cell carcinoma of the vagina.

She had primary vaginal cancer staged as FIGO stage II, and definitive CRT was planned [21]. External beam radiotherapy (EBRT) was given in 28 fractions of 1.8 Gy via intensity-modulated RT (IMRT) technique, with concurrent 6 cycles of $40 \mathrm{mg} / \mathrm{m}^{2}$ weekly cisplatin (Figure 4A). The primary clinical target volume (CTV) included gross tumor volume (GTV), which was expanded by 1-2 $\mathrm{cm}$ margin, entire length of the vagina, and paravaginal tissue. The lymphatic CTV contained the external iliac, internal iliac, presacral, obturator, and inguinal lymph node regions. The CTV was expanded by $1 \mathrm{~cm}$ to form planning target volume (PTV). Bladder, rectum, sigmoid colon, and bowel were delineated as organs at risks
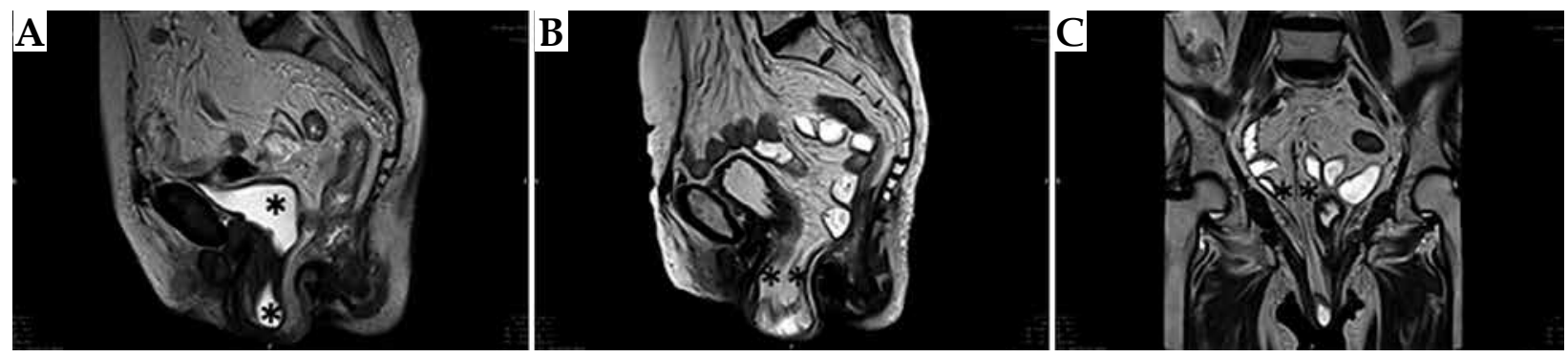

Fig. 2. A) Sagittal T2-weighted (W) image of the pelvic MRI shows cystocele. B, C) Sagittal and coronal T2-weighted (W) images of the pelvic MRI show enterocele with ileal segments and mesentery. All images were obtained in static imaging without Valsalva's maneuver ( ${ }^{*}$ bladder, ${ }^{* *}$ ileal segments, and mesentery)
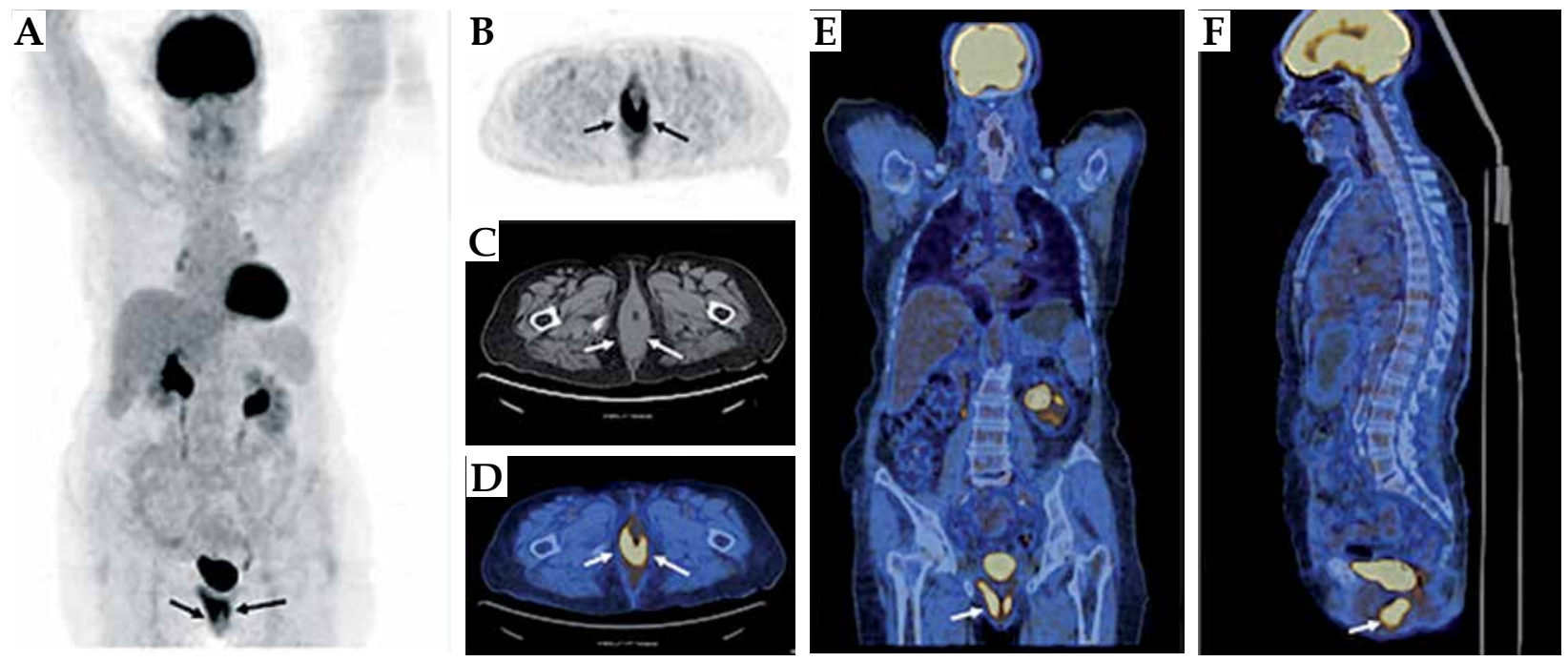

Fig. 3. FDG PET/CT images show increased bilateral uptake in the thickened posterior vaginal wall with an $\mathrm{SUV}_{\max }$ of 16.37 . A) Maximum intensity projection images (MIP). B-F) FDG PET, CT, axial, coronal, and sagittal fusion images (arrows) 

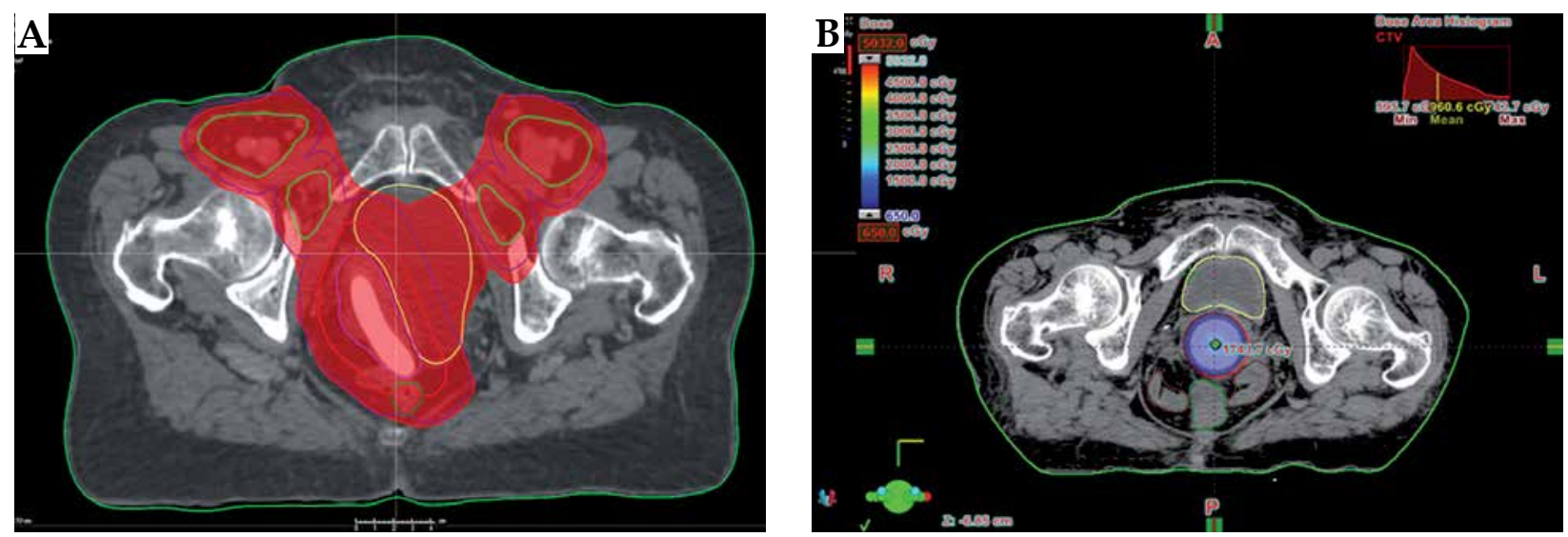

Fig. 4. A) Axial image of CT simulation for radiation therapy and $95 \%$ dose color wash of IMRT treatment planning. B) Axial image of CT simulation for HDR brachytherapy. The gross tumor volume (red line) was well covered by the prescribed $6.5 \mathrm{~Gy}$ isodose line

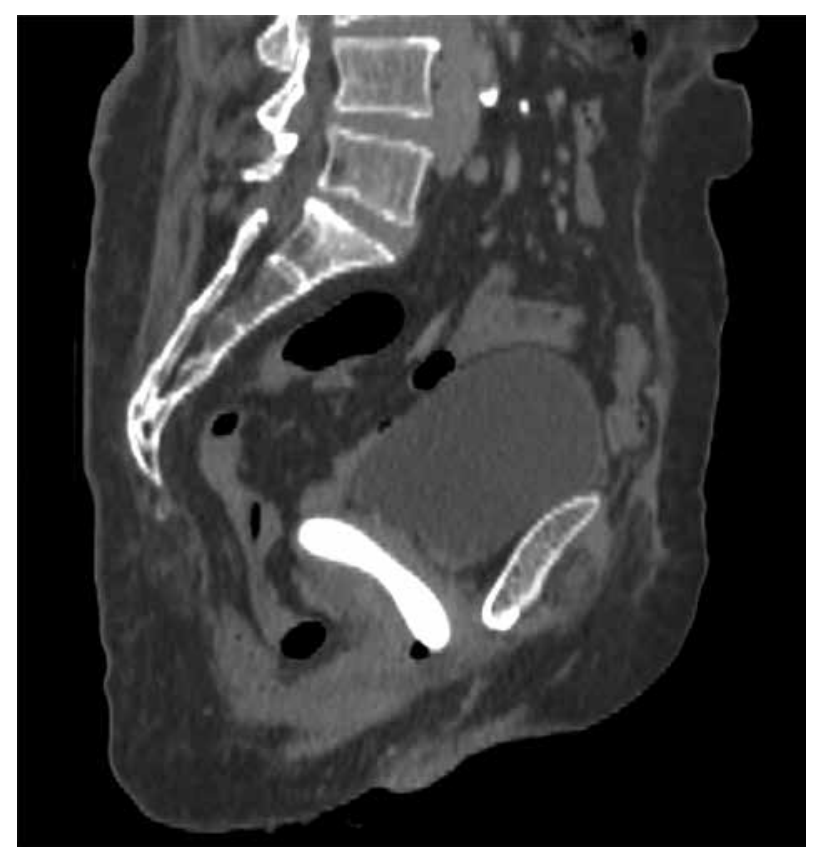

Fig. 5. Sagittal image of computed tomography taken after pessary placement

(OARs). Before EBRT, silicone ring pessary without central support diaphragm (size 3, 2.50" $64 \mathrm{~mm}$ ) was placed into the vagina to help reposition and support the prolapsed vagina, to immobilize the radiation field, and to reduce prolapse symptoms (Figure 5). The pessary was left in place until EBRT was completed. At the end of the EBRT, the pessary was removed. Vaginal examination revealed a complete response (Figure 6). After clinical confirmation of complete response, high-dose-rate (HDR) CT-guided vaginal cuff BT was performed, with a dose of $26 \mathrm{~Gy}$ in 4 fractions prescribed to the full thickness of the vaginal wall (Figure $4 \mathrm{~B})$. Total (EBRT + BT) biologically equivalent dose in 2 Gy fractions $\left(\mathrm{EQD}_{2}\right)$ were calculated with $\alpha / \beta=10$ Gy for tumor and $\alpha / \beta=3$ Gy for late-responding tissues using the linear quadratic model [22]. The total cumulative doses were as follows: primary

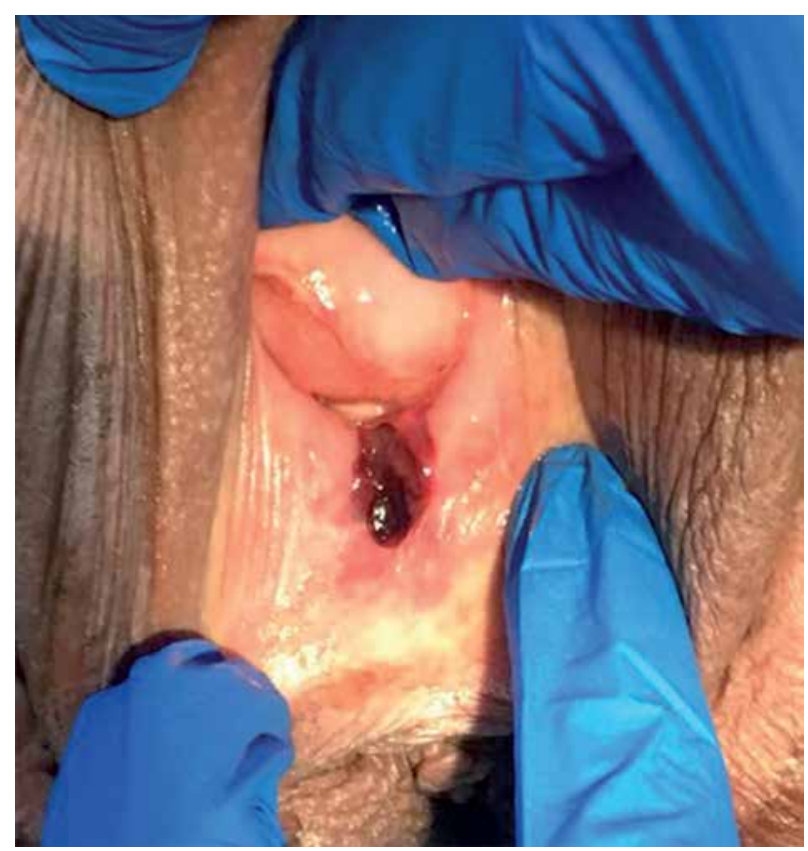

Fig. 6. Appearance of the site of the initial mass with complete response after chemoradiotherapy

$\mathrm{CTV}=85.3 \mathrm{~Gy}$, rectum $\mathrm{D}_{2 \mathrm{~cm} 3}=69.9 \mathrm{~Gy}$, sigmoid $\mathrm{D}_{2 \mathrm{~cm} 3}=$ $57.5 \mathrm{~Gy}$, bladder $\mathrm{D}_{2 \mathrm{~cm} 3}=76.9 \mathrm{~Gy}$.

Treatment was well tolerated. During CRT, grade 3 hematologic toxicity was observed and treated with blood transfusions. Grade 2 dermatitis in the vulva was observed at the end of treatment and was treated with topical agents (Figure 7). The patient had no severe complaints other than mild vaginal pain. At 3-months follow-up, although vaginal examination showed complete response (Figure 8), PET/CT revealed high F-18 FDG uptake in the region of primary vaginal mass $\left(\mathrm{SUV}_{\max }, 5.7\right)$ compatible with partial response (Figure 9). A biopsy was performed to exclude recurrence or residual disease and the complete response was confirmed. She is still alive without disease at 10-months follow-up. However, the patient experienced symptoms like vaginal dryness, itch- 


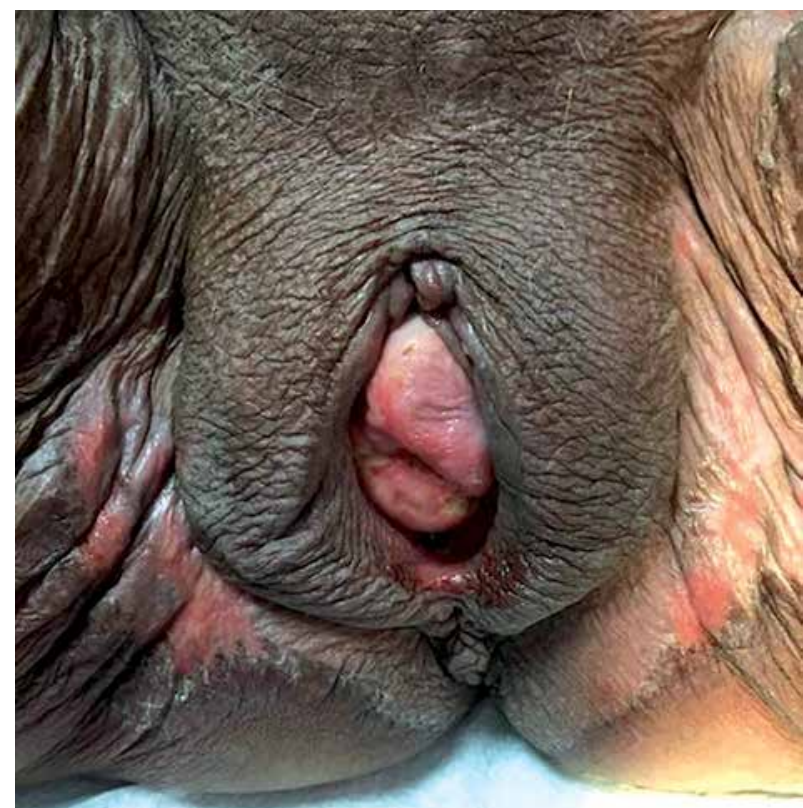

Fig. 7. Grade 2 dermatitis in the vulva after external beam radiotherapy

ing, and burning possibly due to POP and vaginal atrophy. She refused to undergo prolapse surgery consisting of sacrocolpopexy. Ring pessary was placed again into her vagina, and topical estrogen cream was prescribed to alleviate her complaints and to maintain a healthy vaginal epithelium.

\section{Discussion}

Primary vaginal cancer is a rare malignancy and mostly seen in elderly people [1]. The most common risk factors for primary vaginal cancer include human papillomavirus (HPV), history of vulvar intraepithelial neoplasia, immunosuppression, multiple sexual part-

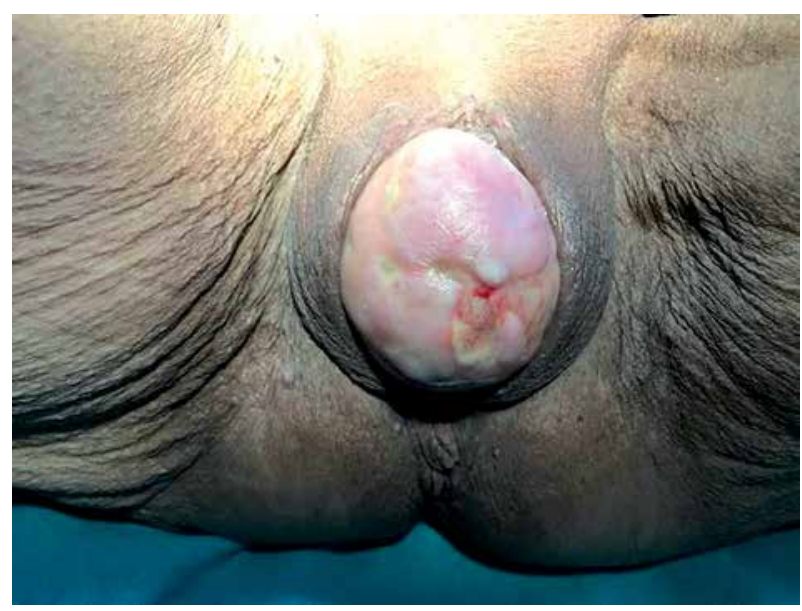

Fig. 8. No residual disease was observed in the physical examination

ners, smoking, early age at first intercourse, low socioeconomic status, history of genital warts, and vaginal trauma $[3,23,24]$. Chronic irritation and inflammation of the vaginal mucosa may also cause primary vaginal cancer.

Pelvic organ prolapse is defined as the descent of female pelvic organs, including the bladder, uterus or post-hysterectomy vaginal cuff, and the small or large bowel [25]. The etiology of prolapse is multifactorial and includes advancing age, increased parity, menopause, collagen weakness, pelvic floor denervation, and direct trauma to the pelvic floor during surgery and deliveries [26]. POP leads to chronic mechanical irritation and adversely affects the quality of life (QoL). Rarely, as seen in our patient, due to chronic irritation, primary vaginal cancer may develop secondary to prolonged POP. However, primary vaginal or cervical cancer and POP combination is rarely reported in the literature $[4,5,6,7,8,9,10,11,12,13,14$, $15,16,17,18,19]$. The increase in the incidence of both POP
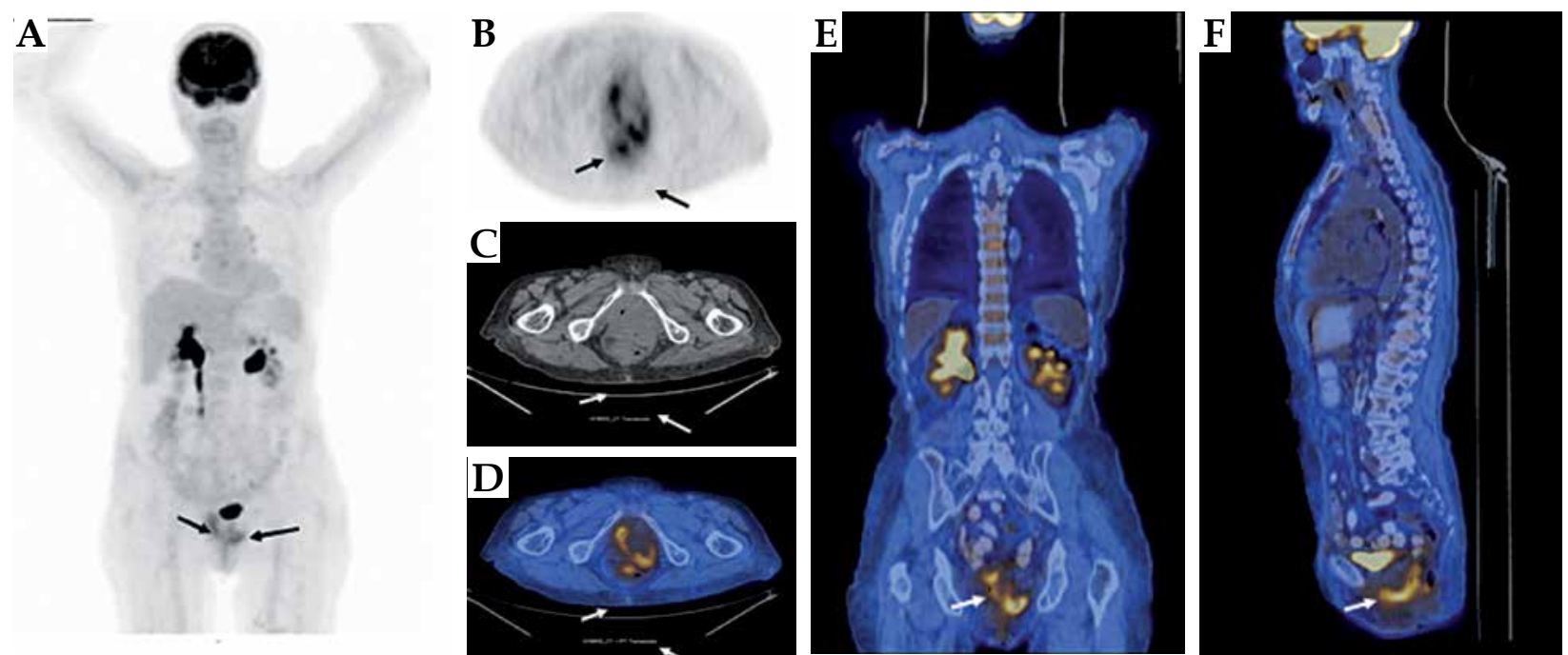

Fig. 9. FDG PET/CT images after chemoradiotherapy showed decreased FDG uptake (SUV max $_{\text {from }} 16.5$ to 5.3$)$ in the vaginal wall compatible with the residual tumor or post-therapy inflammatory changes. A) Maximum intensity projection images (MIP). B-F) FDG PET, CT, axial, coronal, and sagittal fusion images (arrows) 
and primary vaginal cancer with advancing age makes this combination more frequent in elderly people [1].

There are no clear treatment recommendations in combined POP and primary vaginal cancer. When the early stage primary vaginal cancer is combined with POP, surgery becomes the first option instead of RT to cure primary disease and also to restore the anatomy $[4,5,6,7,8,11,12,13]$. Interstitial BT is another treatment option for medically inoperable patients with early-stage disease [19]. However, RT or CRT are the primary choice of treatment for most patients with locally advanced disease, and usually requires careful integration of EBRT and vaginal BT $[6,9,10,27,28]$. When applying EBRT in cases with combined POP and vaginal cancer, critical organ doses may result in larger than expected dose due to the downward descent of the pelvic organs. Additionally, high complication rates in these patients have been reported in the literature $[10,17]$. Rao et al. reported that two of 5 patients developed vesicovaginal fistula following definitive EBRT [10]. Reimer et al. used neoadjuvant CRT and vaginal BT for the cervical cancer patient with irreducible uterovaginal prolapse [17]. Hemorrhagic cystitis could not be avoided after an administered dose of 36 Gy EBRT in this patient.

Pessaries are devices that are placed into the vagina to restore pelvic anatomy and decrease prolapse symptoms [4]. Currently, using of pessary is the only available, non-surgical intervention especially for high-risk surgical candidates with POP, and is reported to relieve prolapse and urinary symptoms, improving health-related QoL. However, using of pessary can also cause serious complications as bleeding and ulceration, and special care should be taken during this treatment [29]. Recently, Dawkins et al. reported a case of 72-year-old woman with cervical cancer with POP, and she was treated with definitive CRT and BT [18]. Gelhorn pessary was placed and perineoplasty was performed before EBRT to reduce $\mathrm{POP}$, and the authors reported no treatment-related side effects.

In our case, definitive CRT was applied, since she had locally advanced disease and she was not suitable for surgery. Before definitive CRT, a ring pessary was placed in order to prevent further increase of POP-related symptoms due to CRT. She tolerated the treatment very well. To our knowledge, presented case is the first case of having definitive CRT with using a pessary who had both vaginal cancer and POP. This treatment approach may limit the risk of genitourinary and gastrointestinal complications due to restoring the anatomy, and reducing radiation exposure to the bladder, rectum, and bowel. It also decreases vaginal irritation and ulceration due to friction during EBRT and may improve patient's QoL. Alternatively, prolapse surgery may be undertaken before CRT, even though the recovery period after surgery may cause delays for definitive treatment. In addition, pessary use seems to be a good option in patients who could not be operated due to comorbid diseases like our patient.

In conclusion, especially in elderly patients, the pessary is well tolerated during definitive CRT, and it is a non-surgical procedure to restore the anatomy for symptom relief and to provide more precise RT.

\section{Disclosure}

The authors report no conflict of intertest.

\section{References}

1. Siegel RL, Miller KD, Jemal A. Cancer statistics, 2016. CA Cancer J Clin 2016; 66: 7-30.

2. Wu JM, Vaughan $\mathrm{CP}$, Goode PS et al. Prevalence and trends of symptomatic pelvic floor disorders in U.S. women. Obstet Gynecol 2014; 123: 141-148.

3. Merino MJ. Vaginal cancer: the role of infectious and environmental factors. Am J Obstet Gynecol 1991; 165: 1255-1262.

4. Iavazzo C, Vorgias G, Vecchini G et al. Vaginal carcinoma in a completely prolapsed uterus. A case report. Arch Gynecol Obstet 2007; 275: 503-505.

5. Karateke A, Tugrul S, Yakut Y et al. Management of a case of primary vaginal cancer with irreducible massive uterine prolapse - a case report. Eur J Gynaecol Oncol 2006; 27: 528-530.

6. Gupta N, Mittal S, Dalmia S et al. A rare case of primary invasive carcinoma of vagina associated with irreducible third degree uterovaginal prolapse. Arch Gynecol Obstet 2007; 276: 563-564.

7. Wang Y, Li Q, Du H et al. Uterine prolapse complicated by vaginal cancer: a case report and literature review. Gynecol Obstet Invest 2014; 77: 141-144.

8. Batista TP, Morais JA, Reis TJ et al. A rare case of invasive vaginal carcinoma associated with vaginal prolapse. Arch Gynecol Obstet 2009; 280: 845-848.

9. Cho MK, Kim CH, Kim YH. Primary invasive carcinoma of the vagina after Le Fort partial colpocleisis for stage IV pelvic organ prolapse: a case report. Int Urogynecol J 2011; 22: 1459-1461.

10. Rao K, Kumar NP, Geetha AS. Primary carcinoma of vagina with uterine prolapse. J Indian Med Assoc 1989; 87: 10-12.

11. Berthelsen HG. Vaginal carcinoma associated with total prolapse; report of a case. Acta Obstet Gynecol Scand 1957; 36: 257-261.

12. Howat JM, Stassan L, Mohandas I et al. Carcinoma of the vagina presenting as a ruptured procidentia with an entero-vaginal fistula and prolapse of the small bowel. Postgrad Med J 1984; 60: 435-436.

13. Ghosh SB, Tripathi R, Mala YM et al. Primary invasive carcinoma of vagina with third degree uterovaginal prolapse: a case report and review of literature. Arch Gynecol Obstet 2009; 279: 91-93.

14. Kim HG, Song YJ, Na YJ et al. A case of vaginal cancer with uterine prolapse. J Menopausal Med 2013; 19: 139-142.

15. Fedus $T$, Raś R, Ksiazek $M$ et al. Primary vaginal squamous cell carcinoma with bladder involvement in uterine prolapsed patient: Case report. Medicine (Baltimore) 2017; 96: e8993.

16. Kriplani A, Relan S, Kumar L et al. Incarcerated procidentia: a rare complication of carcinoma cervix. Aust $\mathrm{N} Z \mathrm{Z}$ Obstet Gynaecol 1995; 35: 463-464.

17. Reimer D, Sztankay A, Steppan I et al. Cervical cancer associated with genital prolapse - a brief review of the literature and long-term results of successful treatment with radiochemotherapy and surgery in a very frail patient. Eur J Gynaecol Oncol 2008; 29: 272-275.

18. Dawkins JC, Lewis GK, Toy EP. Cervical cancer complicating pelvic organ prolapse, and use of a pessary to restore anatomy for optimal radiation: A case report. Gynecol Oncol Rep 2018; 26: 14-16.

19. Ishibashi N, Maebayashi T, Asai-Sato M et al. Radiation therapy for vaginal cancer in complete uterine prolapse with in- 
trauterine adhesion: a case report. BMC Womens Health 2019; 19: 69.

20. Persu C, Chapple CR, Cauni V et al. Pelvic Organ Prolapse Quantification System (POP-Q) - a new era in pelvic prolapse staging. J Med Life 2011; 4: 75-81.

21. FIGO Committee on Gynecologic Oncology. Current FIGO staging for cancer of the vagina, fallopian tube, ovary, and gestational trophoblastic neoplasia. Int J Gynaecol Obstet 2009; 105: 3-4.

22. Dale RG. The application of the linear-quadratic dose-effect equation to fractionated and protracted radiotherapy. Br J Radiol 1985; 58: 515-528.

23. De Vuyst H, Clifford GM, Nascimento MC et al. Prevalence and type distribution of human papillomavirus in carcinoma and intraepithelial neoplasia of the vulva, vagina and anus: a meta-analysis. Int J Cancer 2009; 124: 1626-1636.

24. Schiffman M, Kjaer SK. Chapter 2: Natural history of anogenital human papillomavirus infection and neoplasia. J Natl Cancer Inst Monogr 2003; 31: 14-19.

25. Jelovsek JE, Maher C, Barber MD. Pelvic organ prolapse. Lancet 2007; 369: 1027-1038.

26. Chaliha C, Khullar V. Surgical repair of vaginal prolapse: a gynaecological hernia. Int J Surg 2006; 4: 242-250.

27. Tran PT, Su Z, Lee P et al. Prognostic factors for outcomes and complications for primary squamous cell carcinoma of the vagina treated with radiation. Gynecol Oncol 2007; 105: 641-649.

28. Frank SJ, Jhingran A, Levenback $C$ et al. Definitive radiation therapy for squamous cell carcinoma of the vagina. Int $\mathrm{J} R \mathrm{a}$ diat Oncol Biol Phys 2005; 62: 138-147.

29. Oliver R, Thakar R, Sultan AH. The history and usage of the vaginal pessary: a review. Eur J Obstet Gynecol Reprod Biol 2011; 156: 125-130. 